\title{
Resonant tunneling of light through thin metal films via strongly localized surface plasmons
}

\author{
W. -C. Tan, T. W. Preist, and R. J. Sambles \\ Thin Film Photonics Group, School of Physics, University of Exeter, Stocker Road, Exeter EX4 4QL, UK
}

(Received 31 May 2000)

\begin{abstract}
We present a theoretical model of the optical response of a silver film having narrow-grooved zero-order gratings on both sides. It is found that incident $p$-polarized photons can resonantly tunnel through such a metal film via exciting standing-wave surface plasmon-polariton (SPP) modes localized in the grooves of the two opposite surfaces. This leads to strong transmission peaks in the visible and ultraviolet regions, while for $s$-polarized photons the film acts as a nearly perfect mirror.
\end{abstract}

A flat silver surface is almost a perfect reflector for electromagnetic waves in the visible region, and its application as a mirror has a long history. The optical response of a silver surface can be easily understood by examining its dielectric function, which, as a good approximation, can be described by the Drude model

$$
\epsilon(\omega)=1+\frac{i \tau \omega_{p}^{2}}{\omega(1-i \omega \tau)}
$$

Here $\omega$ is the angular frequency of the radiation, $\omega_{p}$ the plasma frequency, and $\tau$ the relaxation time of the electrons. For silver at room temperature $\omega_{p}=1.32 \times 10^{16} \mathrm{~s}^{-1}$ and $\tau$ $=1.45 \times 10^{-14} \mathrm{~s}^{1}{ }^{1}$ Below $\omega_{p}$ the real part of $\epsilon$ is negative and only evanescent waves are allowed in the metal. The penetration of light into the metal is characterized by the skin depth $\delta=c / 2 \omega \sqrt{\epsilon}$. In the visible region $\left(1.7 \times 10^{16} \mathrm{~s}^{-1}<\omega\right.$ $<3.0 \times 10^{16} \mathrm{~s}^{-1}$ ) the skin depth of silver can be approximately expressed as $\delta \approx c / 2 \omega_{p}=12.5 \mathrm{~nm}$. The transmission coefficient (of intensity) through a flat metal film of thickness $t$ is

$$
T=e^{-t / \delta} \text {. }
$$

Thus a flat silver film with a thickness $t>100 \mathrm{~nm}$ is effectively opaque. It is, in fact, a very good mirror in the visible region because the absorption resulting from the small imaginary part of $\epsilon$ is very weak.

An imposed surface corrugation may, however, significantly alter the optical response of a metal surface, and a corrugated silver surface may no longer be a good mirror. It is very well known that an incident plane wave will be reflected into diffracted orders by a diffractive surface. In addition, a grating can also provide the in-plane momentum required for the incident radiation (if appropriately polarized) to excite a surface plasmon polariton (SPP), resulting in strong optical absorption. This effect has been extensively studied in the last century. ${ }^{2}$ A recent development is the investigation of SPP resonances on zero-order metal gratings, which are gratings with pitches smaller than half the wavelength of the electromagnetic waves considered. It may appear that incident radiation cannot excite SPP modes on a zero-order metal grating because there are no diffracted orders, and subsequently a zero-order grating should still be a good mirror. However, it has been shown recently that because of the narrowness of the grooves of such zero-order gratings, surface charges on the opposite sides of a groove are strongly correlated, leading to the formation of a new family of strongly localized standing-wave SPP modes in the grating grooves. ${ }^{3-6}$ Resonant excitation of these localized SPP modes on zero-order gratings by incident $p$-polarized radiation ( $H$ field parallel to the grating grooves) in the incident plane containing the grating vector results in strong field enhancement and strong frequency-selective absorption. ${ }^{4,6,7}$ Therefore a zero-order grating may be used as an effective frequency-selective absorber for $p$-polarized radiation and, at the same time, a good mirror for $s$-polarized radiation.

Another important recent development in metallic gratings is the study of optical transmission of thin-film metal gratings, which was stimulated by a report of unexpectedly large optical transmission through metallic films with a periodic array of subwavelength size holes by Ebbesen et al. ${ }^{8}$ The motivation of these studies is twofold. First, the effect discovered by Ebbesen and collaborators may find a wide range of potential applications in areas such as optical displays, near-field microscopy, and photolithography. ${ }^{9}$ Second, the understanding of the physical mechanism behind such a strong transmission, which is much larger than expected from diffraction theory ${ }^{10}$ for an isolated hole, poses a theoretical challenge. It has been proposed that the effect is due to the resonant interaction of the incident light with SPP modes in the surfaces of the metal film. ${ }^{9}$ However, the detailed physical picture of the interaction is still not clear.

Very recently, Porto, Garcia-Vidal, and Pendry ${ }^{11}$ studied theoretically a very interesting structure-a metallic grating with very narrow slits. They found that $p$-polarized incident light can excite a series of standing modes in the slits, leading to very large transmission resonances through the grating. This study provides some physical insight into the mechanism of the strong transmission through twodimensional (2D) hole arrays. A numerical study of a metal film with narrow slits was also reported by Schroter and Heitmann. ${ }^{12}$ However there is an important difference between a thin film with an array of subwavelength size holes and a film with subwavelength width slits, which is that a subwavelength width slit can support propagating modes traveling along the direction perpendicular to the film plane while a subwavelength hole can only support evanescent waves along that direction. Therefore the mechanism proposed by Porto et al. is not necessarily directly related to the experiment of Ebbesen et al. ${ }^{8}$ 


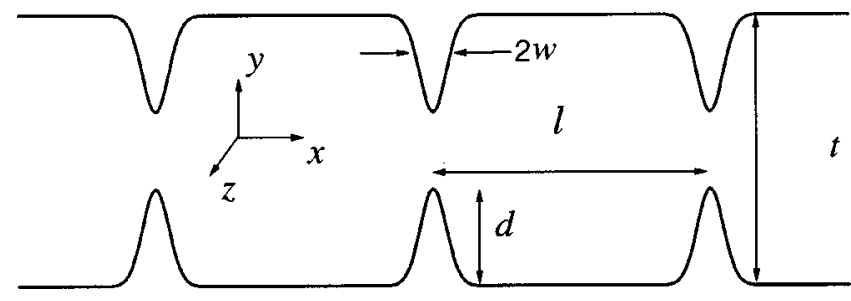

FIG. 1. A schematic illustration of the thin film grating.

In this paper we propose a new mechanism for enhanced optical transmission through thin metallic zero-order gratings by theoretical examination of the structure schematically illustrated in Fig. 1. The model structure is a freestanding thin silver film having periodic Gaussian-shaped grooves on both surfaces which are described mathematically by

$$
\begin{aligned}
& z(x)=t-d \sum_{m=-\infty}^{\infty} \exp \left[-\left(\frac{x-m l}{w}\right)^{2}\right], \text { top surface } \\
& z(x)=d \sum_{m=-\infty}^{\infty} \exp \left[-\left(\frac{x-m l}{w}\right)^{2}\right], \text { bottom surface. }
\end{aligned}
$$

As can be seen in Fig. 1 the profiles of the top and the bottom surfaces are symmetric about the plane $z=t / 2$. Each of the surfaces contains a periodic array of identical Gaussian-shaped grooves. The depth of these grooves, $d$, is taken to be less than $t / 2$, where $t$ is approximately the maximum thickness of the film. We assume that the minimum separation between the grooves on the opposite surfaces is $s=t-2 d>0$; therefore there should be no propagating electromagnetic modes between the top and the bottom surfaces, a situation rather more similar to the hole experiment of Ebbesen et al. than the narrow slits model of Porto et al. The model also has the advantage that the width and depth of the grooves, the grating pitch, and the maximum and minimum of the film thickness can all be controlled easily by adjusting the four parameters $d, w, l$, and $t$, which facilitates the flexibility required for investigation of both photon-plasmon coupling and plasmon-plasmon coupling mechanisms.

Our numerical method is based on a combination of a coordinate transformation technique proposed by Chandezon et al. ${ }^{13}$ and a scattering matrix approach. ${ }^{14}$ It allows the calculation of both reflection and transmission of systems with frequency-dependent permittivities. In our calculation the Drude model is used to describe the dielectric function of silver. We assume that both the top and bottom media are air, which has a frequency-independent optical permittivity $\epsilon$ $=1.0006$.

Figure 2 shows the calculated transmission coefficient $\left(T_{p p}\right)$ and reflection coefficient $\left(R_{p p}\right)$ as a function of frequency for normally incident $p$-polarized radiation $(H$ field parallel to the grooves) on the top side of several silver films. For ease of analysis, in our calculation, we assume that all the silver films have the same surface profile, which is defined by $l=150 \mathrm{~nm}, d=50.5 \mathrm{~nm}$, and $w=10 \mathrm{~nm}$, while their thickness $t$ is varied from $102 \mathrm{~nm}$ to $251 \mathrm{~nm}$ (only the results for $t \leqslant 161 \mathrm{~nm}$ are presented). As can be seen from Fig. 2(a), in the transmission coefficient curve of the $t=161 \mathrm{~nm}$ film,
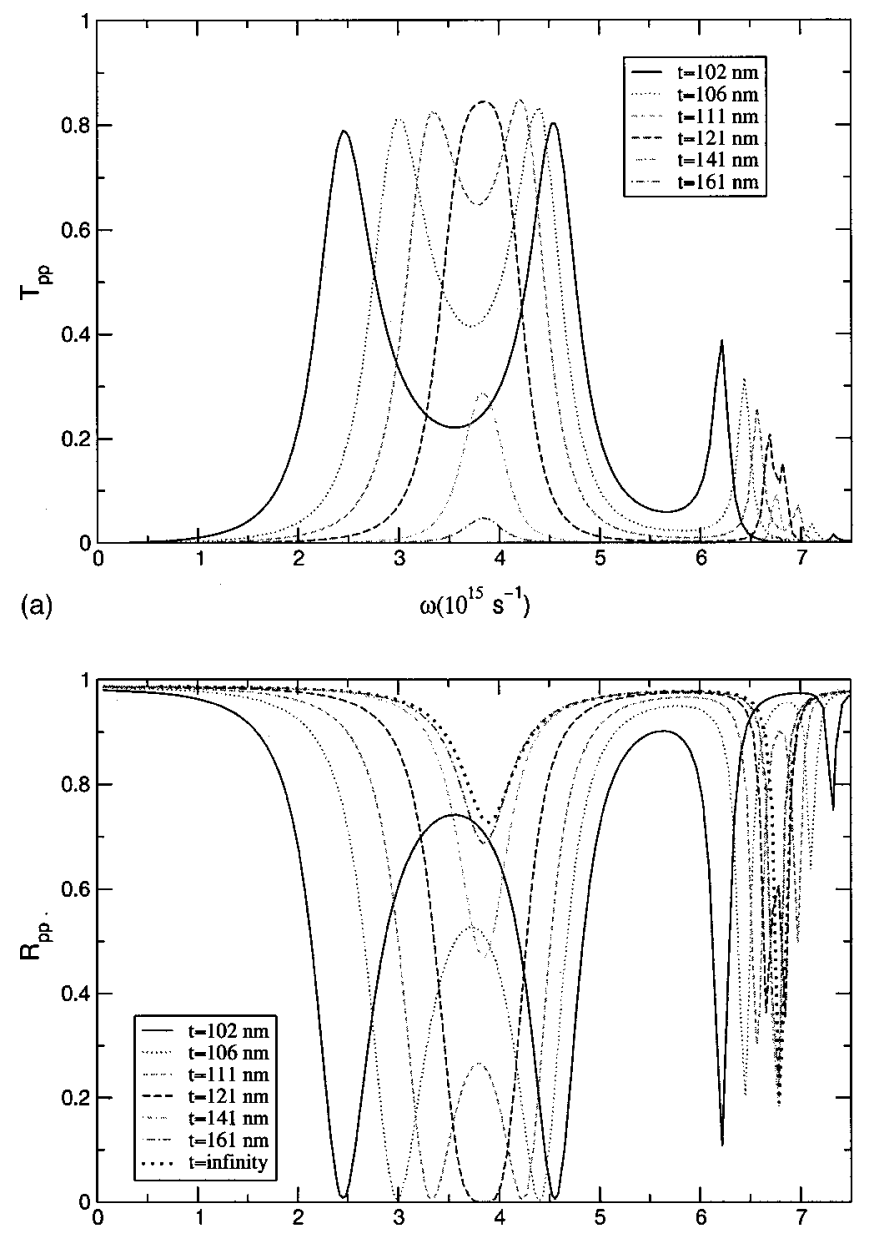

(b)

$\omega\left(10^{15} s^{-1}\right)$

FIG. 2. (a) Transmission and (b) reflection coefficients as a function of frequency for normally incident $p$-polarized radiation calculated for silver films with the same surface profile $(l=150 \mathrm{~nm}$, $d=50.5 \mathrm{~nm}$, and $w=10 \mathrm{~nm}$ ) but different film thickness as marked.

there are two small peaks at frequencies $\omega_{1} \approx 3.83 \times 10^{15} \mathrm{~s}^{-1}$ (green) and $\omega_{2} \approx 6.72 \times 10^{15} \mathrm{~s}^{-1}$ (ultraviolet). With a small decrease of film thickness the two transmission peaks become more prominent, and their positions remain unchanged. For the $t=121 \mathrm{~nm}$ film, the transmission coefficient at the first peak is about 0.85 . On further decrease of $t$, each of the two transmission peaks splits into two. For the film with $t$ $=102 \mathrm{~nm}$ we see that there are two well-separated transmission peaks in the infrared and violet regions, respectively.

As expected Fig. 2 shows that a peak in a transmission curve always corresponds to a minimum in the reflection curve at the same frequency. It should be pointed out that these strong transmission peaks only occur for $p$-polarized incident radiation. Our calculation shows that, for $s$-polarized incident radiation, the transmission coefficient can be approximately described by Eq. (2) and the existence of the grating grooves has little effect on the transmission of $s$-polarized waves. For example, at $\omega_{1} \approx 3.83 \times 10^{15} \mathrm{~s}^{-1}$ the transmission coefficient of $s$-polarized radiation through the $t=121 \mathrm{~nm}$ film is $T_{s s}=0.00016$. This shows that the grating grooves on the film surface have enhanced the transmission for $p$-polarized radiation by a factor of $T_{p p} / T_{s s}=5.5 \times 10^{3}$.

To understand the physical origin of the enhanced transmission we start by looking at very thick films. For a film 

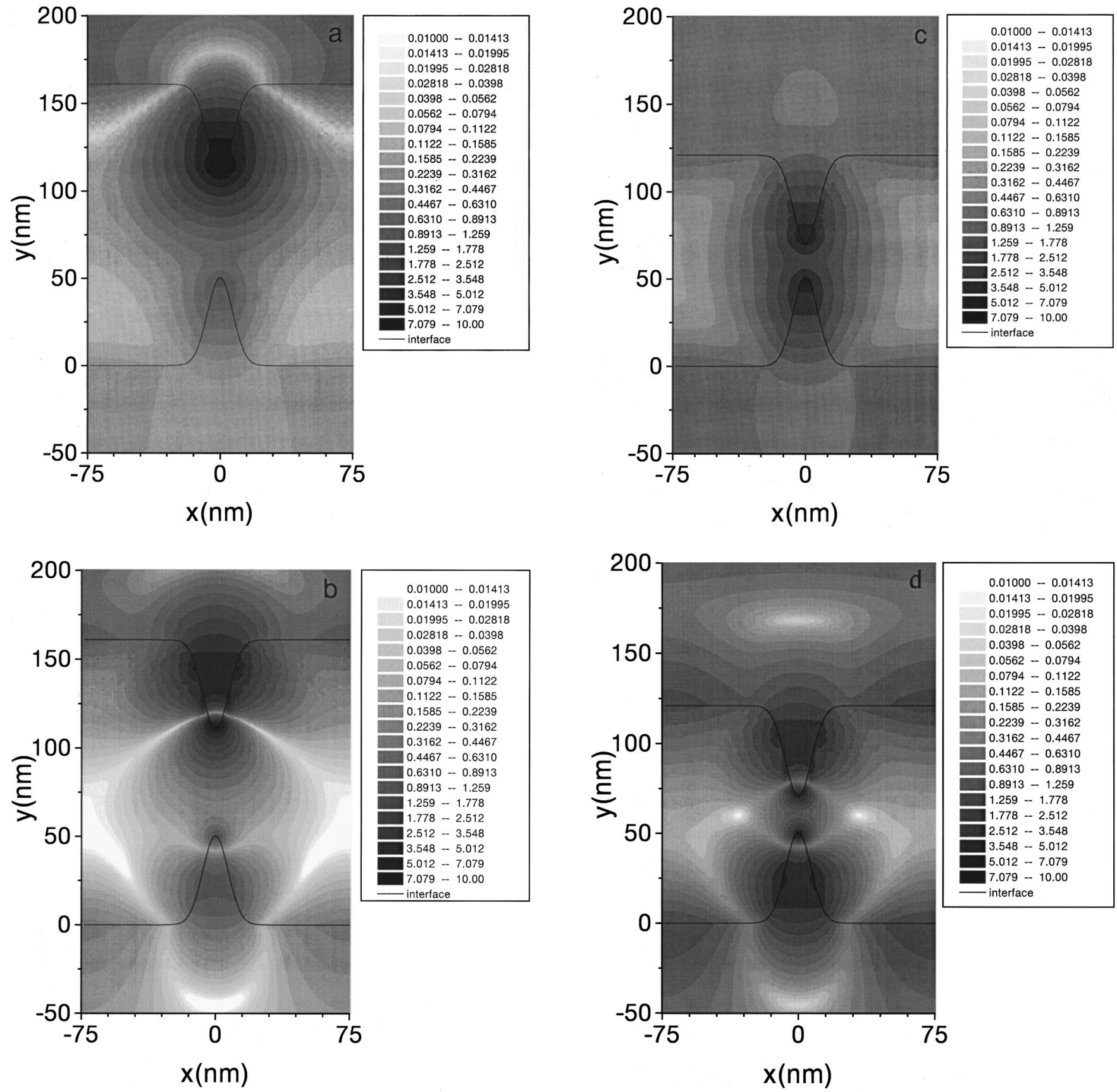

FIG. 3. Distribution of $\left|H_{z}\right|$ for various resonances. (a) $t=161 \mathrm{~nm}, \omega=3.86 \times 10^{15} \mathrm{~s}^{-1}$; (b) $t=161 \mathrm{~nm}, \omega=6.67 \times 10^{15} \mathrm{~s}{ }^{-1}$; (c) $t$ $=112 \mathrm{~nm}, \omega=3.86 \times 10^{15} \mathrm{~s}^{-1}$; (d) $t=112 \mathrm{~nm}, \omega=6.67 \times 10^{15} \mathrm{~s}^{-1}$; (e) $t=102 \mathrm{~nm}, \omega=2.45 \times 10^{15} \mathrm{~s}^{-1}$; (f) $t=102 \mathrm{~nm}, \omega=4.52 \times 10^{15} \mathrm{~s}^{-1}$.

thickness $t \rightarrow \infty$, the incident radiation cannot "sense" the bottom surface and the reflection coefficient curve should be identical to that of a single air/silver interface, which has two deep minima at $\omega_{1} \approx 3.83 \times 10^{15} \mathrm{~s}^{-1}$ and $\omega_{2} \approx 6.72 \times 10^{15}$ $\mathrm{s}^{-1}$. Since there are neither transmitted waves nor diffracted orders, any deviation of the reflection coefficient from one must be due to absorption. The optical response of zeroorder silver gratings containing an array of narrow Gaussian grooves has been analyzed in detail in Ref. 6. Following Ref. 6 , in both the top and the bottom surface there should be a series of standing-wave SPP modes strongly localized in the grooves. Resonant excitation of a localized SPP mode in the top surface grooves results in strong field enhancement and a reflection minimum at the frequency of the SPP mode.
For a film with a finite thickness, when the incident radiation matches the frequency of a standing-wave SPP mode, it resonantly excites the SPP mode on the top surface and builds up strong fields in the grooves. This in turn resonantly excites the standing-wave SPP mode on the bottom surface. A strong EM field is then built up in the grooves of the bottom surface, which finally emits the radiation downwards into the air. Therefore resonant transmission happens at the frequencies of standing-wave SPP modes. This transmission process is effectively photon tunneling through a metal film via resonantly exiting localized SPP modes. Clearly such resonant transmission cannot occur for $s$-polarized radiation since it cannot couple to the SPP modes. This mechanism can be readily seen from the optical magnetic field distribu- 

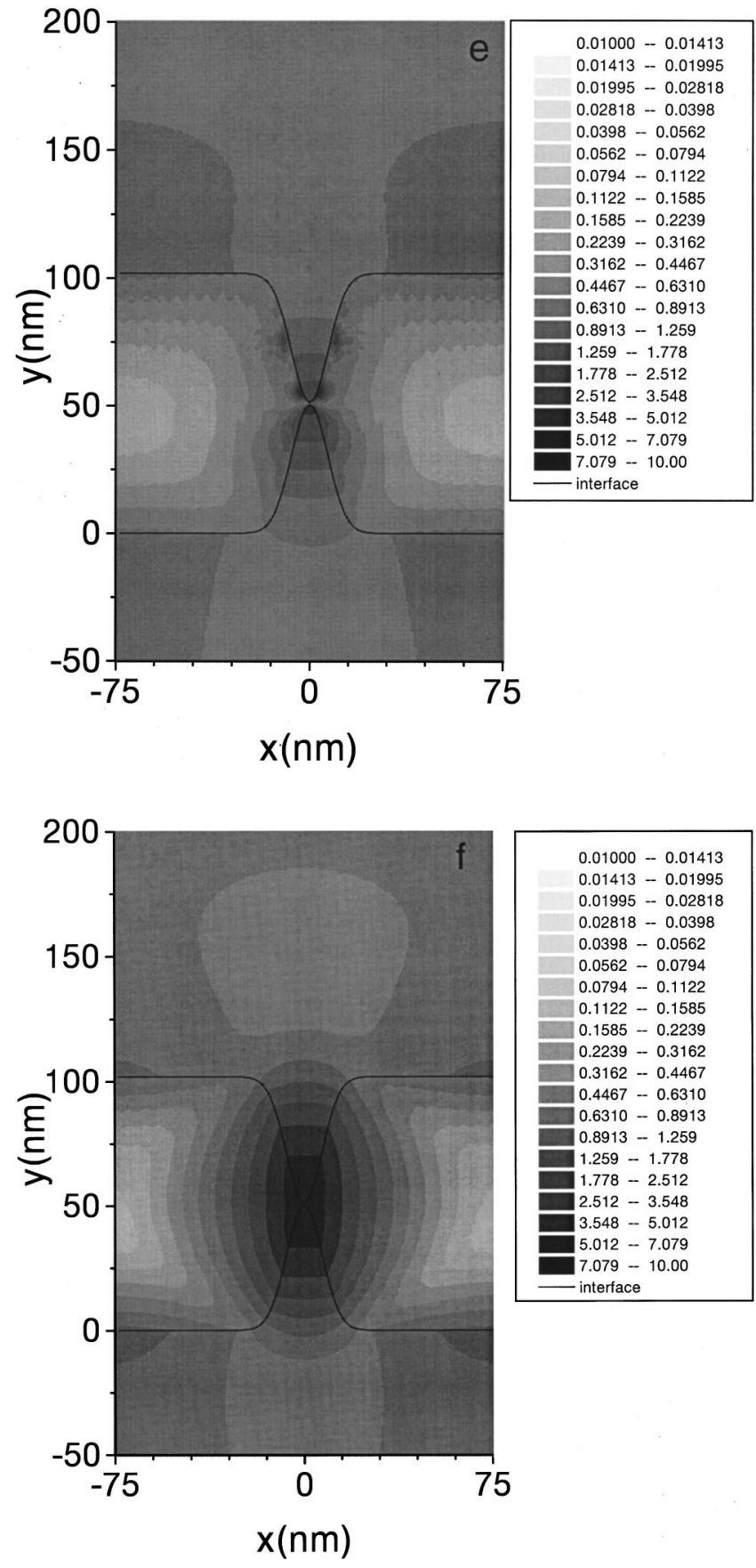

FIG. 3. (Continued.)

tions given in Figs. 3(a) and 3(b), which, respectively, show the resonant excitation of the first two standing-wave SPP modes by incoming radiation.

The width of a standing-wave SPP mode with a frequency $\omega_{s p p}$ on the top surface of a metal film may be approximately characterized by just two parameters, $\Delta \omega_{\text {ra }}$, the frequency broadening due to the coupling to the free radiation in the space above the film, and $\Delta \omega_{t b}$, the frequency broadening due to the coupling to the standing-wave SPP mode on the bottom surface. For the moment we ignore the thermal broadening of the mode due to absorption of the metal. $\Delta \omega_{r a}$ is mainly determined by the surface profile and the dielectric constant of the metal. $\Delta \omega_{t b}$ is proportional to $f^{2} e^{-s / \delta}$, where $f$ is the field enhancement factor of the SPP mode and $s$ the minimum thickness of the film. For a thick film in which $\Delta \omega_{t b} \ll \Delta \omega_{r a}$, the transmission coefficient at resonance is proportional to $\Delta \omega_{t b}$. Therefore the height of a transmission peak increases exponentially as the film thickness decreases, as can be seen in Fig. 2(a). When $\Delta \omega_{t b}=\Delta \omega_{r a}$, the height of the peak should reach a maximum value of 1 (less than 1 in practice because of the absorption). At this condition, the standing-wave SPP modes on both surfaces are nearly equally excited and the distribution of field amplitudes on both surface are almost symmetric, as is shown in Figs. 3(c) and $3(\mathrm{~d})$.

With further decrease of the film thickness, the separation between the bottoms of the grooves on the two opposite surfaces becomes very small, and we have $\Delta \omega_{t b}>\Delta \omega_{r a}$. In this regime, it is no longer a good approximation to consider the SPP modes on each surface as individual entities. The strong coupling between the two SPP modes on the opposite surfaces creates a pair of new modes with a frequency separation proportional to $\Delta \omega_{t b}$. The frequency splitting of the two SPP modes is rather like the energy splitting of electron states in a symmetric double-quantum-well structure in semiconductor physics when the barrier between the two wells decreases. Exciting each of these two modes by incident radiation will result in a resonant transmission peak, which is what we see in Fig. 2. The pair of SPP modes has different symmetries. As is shown in Fig. 3(e), the magnetic field distribution of the low-frequency mode is antisymmetric about the central line of the film $z=t / 2$ with a node (in practice a minimum) at $z=t / 2$, while that of the highfrequency mode, Fig. 3(f), is symmetric about $z=t / 2$ with a maximum at $z=t / 2$.

Now let us analyze how various physical parameters affect the amplitude and line shape of a resonant transmission peak. A metallic thin film grating may be regarded as a switch, which redirects an incident photon into different channels: transmission, zero-order reflection, high diffractive order reflections, and absorption. To optimize the transmission we need to minimize the probabilities to the remaining channels. Clearly the most obvious ingredient is to make the grating of zero order so all the diffraction channels are eliminated, which is exactly the reason why in the present work we consider zero-order gratings.

For a SPP resonance on a zero-order grating, the probability of an incident photon being scattered into one of the three available channels (zero-order reflection, transmission, and absorption) depends on the coupling strength between the SPP mode in the film and the given channel. For the silver films considered here the SPP modes are strongly localized; therefore their coupling to the reflection and transmission channels are weak. As a good approximation, the transmission, absorption, and reflection coefficients in the vicinity of a SPP resonance at $\omega_{s p p}$ may be expressed as

$$
\begin{gathered}
T=\frac{\Delta \omega_{r a} \Delta \omega_{r b}}{\left(\omega-\omega_{s p p}\right)^{2}+\Delta \omega_{s p p}^{2} / 4}, \\
T_{a b}=\frac{\Delta \omega_{r b} \Delta \omega_{r b}}{\left(\omega-\omega_{s p p}\right)^{2}+\Delta \omega_{s p p}^{2} / 4}, \\
R=1-T-T_{a b},
\end{gathered}
$$


where $\Delta \omega_{s p p}=\Delta \omega_{r a}+\Delta \omega_{r b}+\Delta \omega_{a b}$ is the total width of the SPP mode, while $\Delta \omega_{r a}, \Delta \omega_{r b}, \Delta \omega_{a b}$ are the widths of the SPP mode due to its coupling to the reflected channel, transmitted channel, and the absorption, respectively. It is easy to see that the conditions for a total transmission are $\Delta \omega_{a b}$ $=0$ and $\Delta \omega_{r a}=\Delta \omega_{r b}$. The three widths are determined by the film thickness, surface profiles, and the dielectric function of the metal film. By choosing an appropriate combination of these parameters, one will be able to obtain specified transmission line shapes.

It should be pointed out that since the standing-wave SPP modes are strongly localized in the grooves, they form very flat bands. As a result the frequencies of the SPP resonances are nearly independent of the angle of incidence. Therefore the metal films considered here have potential application as optical filters. They may also be used as polarizers because only $p$-polarized photons may tunnel through the films while $s$-polarized photons are effectively blocked. Another important feature of the thin film grating proposed in this work is that the wavelength of a standing-wave SPP mode in the narrow grooves is much shorter than the optical wavelength at the same frequency. Consequently resonant photon transmission can occur even when the wavelength of the photons is much larger than the thickness of the metal film, as shown in Fig. 2. This effect will be useful for novel device applications, especially in the area of integrated optoelectronic systems where the size of the components is vital.

In conclusion we have proposed an alternative mechanism for strong optical transmission through metallic films. By modeling a silver film, which has an array of narrow grooves on both surfaces, we demonstrate that, via resonantly exciting the standing-wave SPP modes trapped in the grooves of both surfaces, $p$-polarized photons may tunnel through the film with a probability close to 1 , while $s$-polarized photons will be almost completely reflected by the film. We have analyzed the physical parameters that affect the amplitude and line shape of the resonant transmission. We believe our results not only shed light on the physical mechanism of the unexpected strong light transmission through metal films having an array of subwavelength size holes observed by Ebbesen et al. ${ }^{8}$ but they may also lead to novel device applications.

\section{ACKNOWLEDGMENTS}

The authors are grateful to the Leverhulme Trust and BBSRC (U.K.) for supporting this research.
${ }^{1}$ D.J. Nash and J.R. Sambles, J. Mod. Opt. 43, 81 (1996).

${ }^{2}$ H. Raether, Surface Plasmons (Springer, New York, 1988); A. A. Maradudin, in Surface Polaritons, edited by V. M. Agranovich and D. L. Mills (North-Holland, New York, 1982), p. 405.

${ }^{3}$ F.J. Garciá-Vidal and J.B. Pendry, Phys. Rev. Lett. 77, 1163 (1996).

${ }^{4}$ M.B. Sobnack, W.C. Tan, N.P. Wanstall, T.W. Preist, and J.R. Sambles, Phys. Rev. Lett. 80, 5667 (1998).

${ }^{5}$ T. Loṕez-Rios, D. Mendoza, F.J. Garciá-Vidal, J. SańchezDehesa, and B. Pannetier, Phys. Rev. Lett. 81, 665 (1998).

${ }^{6}$ W.-C. Tan, T.W. Preist, J.R. Sambles, and N.P. Wanstall, Phys. Rev. B 59, 12661 (1999).

${ }^{7}$ W.-C. Tan, J.R. Sambles, and T.W. Preist, Phys. Rev. B 61, 13
177 (2000).

${ }^{8}$ T.W. Ebbesen, H.J. Lezec, H.F. Ghaemi, T. Thio, and P.A. Wolff, Nature (London) 391, 667 (1998).

${ }^{9}$ J.R. Sambles, Nature (London) 391, 641 (1998); P.R. Villeneuve, Phys. World 11, 28 (1998); T.J. Kim, T. Thio, T.W. Ebbesen, D.E. Grupp, and H.J. Lezec, Opt. Lett. 24, 256 (1999).

${ }^{10}$ H.A. Bethe, Phys. Rev. 66, 163 (1944).

${ }^{11}$ J.A. Porto, F.J. Garcia-Vidal, and J.B. Pendry, Phys. Rev. Lett. 83, 2845 (1999).

${ }^{12}$ U. Schroter and D. Heitmann, Phys. Rev. B 58, 15419 (1998).

${ }^{13}$ J. Chandezon, M.T. Dupuis, G. Cornnet, and D. Maystre, J. Opt. Soc. Am. 72, 839 (1982).

${ }^{14}$ D.Y.K. Ko and J.R. Sambles, J. Opt. Soc. Am. A 5, 1863 (1988). 